\title{
Influence of sample size on flatness estimation and uncertainty in three-dimensional measurement
}

\author{
A. Jalid ${ }^{1, \star}$, S. Hariri ${ }^{2}$, and N.E. Laghzale ${ }^{1}$
}

1 Laboratoire de recherche (LaMIPI), Université Mohammed V- Rabat (UM5R), ENSET de Rabat, Avenue de l'Armée Royale, Madinat Al Irfane, 10100 Rabat Maroc

2 Département Technologie des Polymères et Composites et Ingénierie Mécanique, Ecole des Mines de Douai, 764 Boulevard Lahure, 59500 Douai, France

Received: 14 November 2014 / Accepted: 12 January 2015

\begin{abstract}
To respond correctly to its function in a mechanism, a mechanical part should have a good dimensional and geometric quality. In three-dimensional measurement, the preferred tool of control is currently the coordinate measuring machine CMM, the evaluation of flatness defect of a part is a very common task in this area, and the decisions made based on the measurement result may be outliers when the uncertainty of measurement result is not taken into account. The evaluation of uncertainty is a delicate task in this area given the diversity of parameters that relies in. The number of points collected on the surface is one of the influencing factors. The choice of this parameter is left to the operator, and the value taken by this parameter affects both the estimated flatness and the associated uncertainty. In this paper we present the effect of a number of points collected during the inspection of a surface on the estimated flatness and the associated uncertainty. A new identification method is presented here. The flatness deviation uncertainty is quantified by applying the law of propagation of uncertainties. The treated data sets are the result of experimental measurements performed on a CMM by measuring an industrial part.
\end{abstract}

Keywords: CMM uncertainties, flatness and uncertainty, effect of sampling flatness

\section{Introduction}

The flatness control of a mechanical part is a very common task in mechanical engineering. The Coordinate measuring machine (CMM) is the control tool used in the field. These machines have a simple principle. That is to say, a sensor mounted at the end of three mechanical guiding systems can probe the points belonging to the geometric element to control; software processing of the data sets to estimate the flatness default of this element. The decisions made from the measurement result may be outliers when the associated uncertainty to the measurement result is not taken into account.

The evaluation of uncertainty on a CMM is a delicate task given the diversity of parameters that relies in. The number of points collected on a flat surface is one of the influencing factors. The choice of this parameter is left at the initiative of the operator, and the value taken by this parameter greatly affects the estimated flatness and the associated uncertainty.

Many studies have been interested in this field of research. For instance, Bourdet [1], Mathieu et al. [2] proposed a method based on the hypothesis of small

\footnotetext{
^ Correspondence: jalid.abdel@gmail.com
}

displacements torsor to estimate the flatness deviation, Carr et al. [3], who estimate the minimum zone solution of a non-linear optimization problem, proposed an algorithm to solve a sequence of linear programs that converge to the solution of the nonlinear problem. Kanada et al. [4] presented nonlinear optimization techniques for minimum zone flatness. The techniques namely used the downhill simplex method and the repetitive bracketing method. The result obtained is compared to the leastsquares method.

Raghunandan et al. presented in [5] the influence of surface quality (i.e. surface roughness) in determining the sampling strategy for accurate determination of flatness error. Moreover, in [6] the authors seek the optimum sample size. Huang presented in [7] a method that solves the straightness and flatness during the construction of the convex hull, which is a tedious job when the number of points is large, and proposes to reduce this number. Gapinski presented in [8] the effect of the sample size on the parameters of the associated feature with applying different criteria and offers a minimum number of points to choose the deviation form. Samuel and Shunmugam [9] developed algorithms based on computational geometric techniques for minimum zone and function-oriented evaluation of straightness and flatness. 

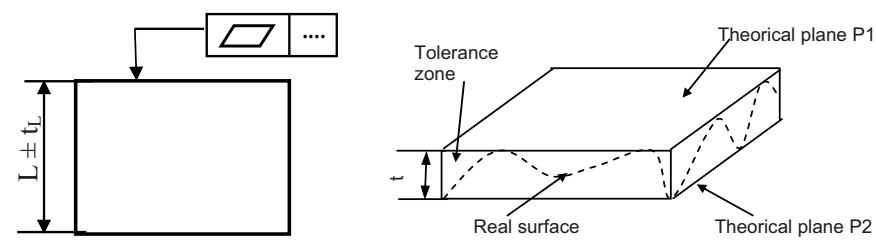

Fig. 1. Flatness deviation.

The studies presented assume that the coordinates of the probed points are not affected with error. This is not the case on a CMM since these studies are limited to the estimation of flatness error without providing the associated uncertainty. They are not in conformity with the requirement of the normative guidelines [10].

In this paper, we propose a new method to estimate the form error and the associated uncertainty that caused by the measuring process, and analyze the effect of sample size to determine the optimum value which meets the requirement of measurement uncertainty. Finally an experimental study dealing with the inspection of flatness on a Coordinate Measuring Machine is proposed.

\section{Flatness error in Norm}

In the ISO 1101 [11] standard, flatness is defined as the minimum distance between two parallel planes $P 1$ and $P 2$ containing all data points (Fig. 1).

The ISO 6318 provides two criteria to evaluate the flatness error, The Least squares criterion (LSC) and the Minimum zone criterion (MZC). The least square criterion is widely used in the industry for assessing the dimensions and geometric tolerances. It is simple to compute, easy to implement in computer processing, and provides unique solutions.

The estimated value of flatness depends on the number and position of the selected points on the part. The choice of sampling strategy is left to operator, the sample size and location of taken points affects both the estimated flatness and the associated uncertainty.

\section{Problem modeling}

\subsection{Parameters of a plane in 3D}

$P$ is a plane, the normal unit vector $\vec{n}\left(n_{x}, n_{y}, n_{z}\right)^{T}$, and $\mathrm{A}$ is a point belonging to a plane. The set of points $M\left(x_{M}, y_{M}, z_{M}\right)^{T}$ belonging to the plane $P$ as shown in Figure 2 verifies:

$A \vec{M} \cdot \vec{n}=0$. This results in:

$\left\{\begin{array}{l}\left(x_{M}-x_{A}\right) \cdot n_{x}+\left(y_{M}-y_{A}\right) \cdot n_{y}+\left(z_{M}-z_{A}\right) \cdot n_{z}=0 \\ \text { with } n_{x}^{2}+n_{y}^{2}+n_{z}^{2}=1\end{array}\right.$

From collected points on the plane $P_{i}\left(x_{i}, y_{i}, z_{i}\right)^{T}$, the $x_{i}, y_{i}, z_{i}$ coordinates are considered as random variables distributed according to a normal distribution with $\sigma_{i}$ as

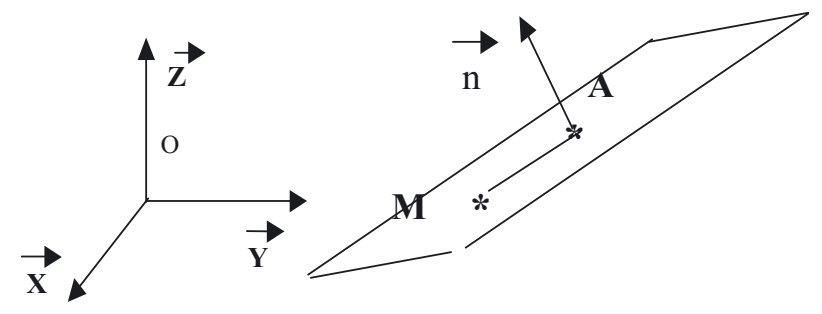

Fig. 2. Parameters of a plane.

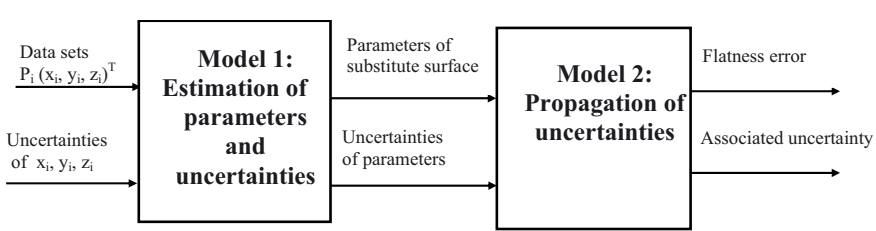

Fig. 3. Input and outputs of the studied models.

a standard deviation. The proposed method used to estimate the parameters has led us to treat a linear optimization problem with stochastic variables; this approach is shown in Figure 3.

This figure shows the inputs and outputs of the studied models. Model 1 estimated from the collected cloud points, the parameters of the substitute feature, and the associated uncertainties. The method used is based on the orthogonal distance regression and will be presented in Section 3.2. A more detailed study shows the development of the model for different geometries (see Jalid et al. [12]). The second model allows us to estimate the flatness error and the associated uncertainty from the parameters and their uncertainties.

\subsection{Estimation of parameters by orthogonal distance regression (Model 1)}

In dimensional metrology and particularly on a CMM, it is assumed that all variables $x_{i}$ and $y_{i}$ are respectively assigned with error $\delta \in R^{1}$ and $\varepsilon \in R^{1}$. The representative model is written as follows:

$$
y_{i}=f\left(x_{i}+\delta_{i} ; \beta\right)-\varepsilon_{i}
$$

The problem is to find the parameters $\beta$ that minimize the sum of squared distances orthogonal to the measured points and the theoretical model as shown in Figure 4.

With $r_{i}$ distance is from the measured point $P_{i}\left(x_{i}, y_{i}\right)$ to the desired model. The coordinate's points are random, assumed and distributed according to a normal distribution with standard deviation $\sigma_{i}$. Moreover, $\omega_{i}=\frac{1}{\sigma_{i}^{2}}$ is a weighting introduced to compensate the case where the measurement accuracy is not the same for all points.

Generalized to the case of several explanatory variables, $x_{i} \in R^{m}$, with $m=3$, has several functions and modeled output $f_{i}: R^{p+m} \rightarrow R^{q}$, with $p$ as the number of parameters $\beta$ and $q$ as the number of functions $f$. 


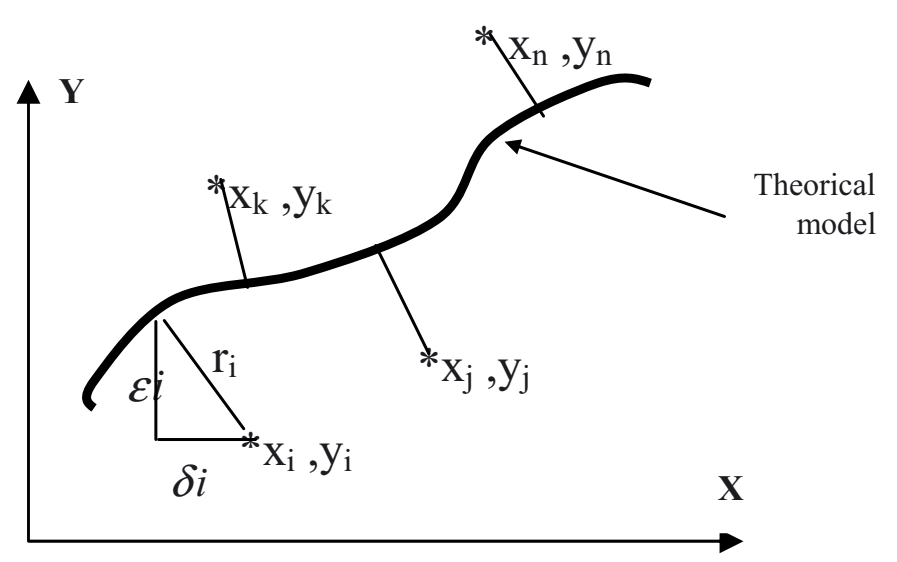

Fig. 4. Orthogonal distance regression.

The problem to solve is then expressed by the following equation:

$$
\left\{\begin{array}{l}
\min _{\beta, \delta} \frac{1}{2} \sum_{i=1}^{n}\left[\delta_{i}^{T}\right]\left[\omega_{\delta_{i}}\right]\left[\delta_{i}\right] \\
\text { with constraints: } \\
f 1_{i}\left(x_{i}+\delta_{i} ; \beta\right)=0 \\
f 2_{i}\left(x_{i}+\delta_{i} ; \beta\right)=0 \\
f q_{i}\left(x_{i}+\delta_{i} ; \beta\right)=0
\end{array} \quad i=1, \ldots, n\right.
$$

where

$\left[\delta_{i}\right]$ : The vector where the elements represent the variations according to $x_{i}$ between the data points and the corresponding points on the fitted model.

$\left[\omega_{\delta_{i}}\right]$ : The matrix of weights of order $(m, m)$.

$\left[f q_{i}\right]$ : The value given by the equation $q$ to the $i$ th observation.

The solution of the problem is iteratively found using a trust region Levenberg-Marquardt method; see Levenberg [13], Marquard [14] and Nocedal [15]. The Jacobian matrices, i.e., the matrices of first partial derivatives of

$$
f_{l i}\left(x_{i}+\delta_{i} ; \beta\right), \quad l=1, \ldots, q, \text { and } i=1, \ldots, n
$$

with respect to each component of $\beta$ and $\delta$, are computed at every iteration by either finite differences.

The parameters' uncertainties are then expressed from the variances of parameters $\beta$. The algorithm of this method is implemented in ODRPACK, Boggs et al. $[16,17]$

After convergence one notes by $G(\eta)$ the vector of the residues, where the ith element is defined by the following:

$$
G_{i}(\eta)=\left\{\begin{array}{lr}
f_{i}\left(x_{i}+\delta_{i} ; \beta\right) \quad i=1, \ldots, n \\
\delta_{i-n} \quad i=n+1, \ldots, 2 n
\end{array}\right.
$$

The matrix of variance covariance of the estimated parameters is given by the equation below:

$$
\sigma^{2}=\left[G(\hat{\eta})^{T} \Omega G(\hat{\eta})\right] /(n-p)
$$

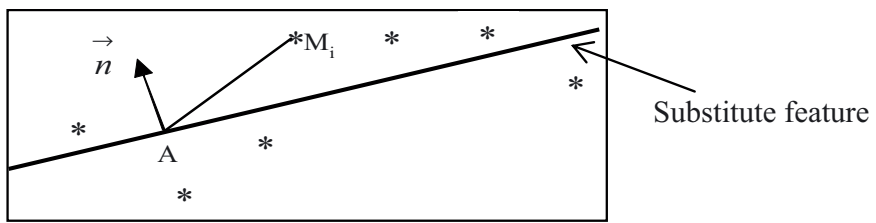

Fig. 5. Parameters of a substitute feature.

where $\Omega$ is the diagonal weighting matrix given by the following:

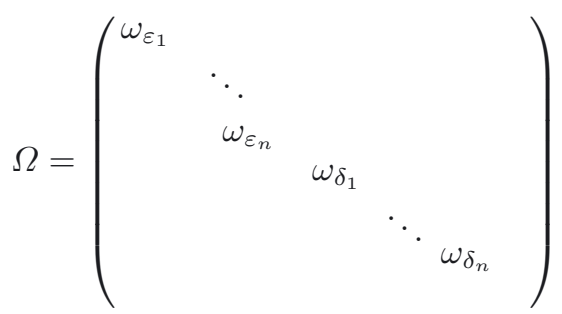

The matrix of the variances and covariances is finally expressed in the form

$$
\hat{V}=\left(\begin{array}{ll}
\hat{V}_{\beta} & \hat{V}_{\beta \delta} \\
\hat{V}_{\delta \beta} & \hat{V}_{\delta}
\end{array}\right)
$$

with $\hat{V}_{\beta}$ the most useful matrix in practice, this matrix is of order $(p, p)$ whose diagonal terms represent the variances of the parameters $\beta$, and the non-diagonal terms represent covariances between the parameters.

$$
\hat{V}_{\beta}=\left(\begin{array}{lll}
\operatorname{var}\left(\beta_{1}\right) & \operatorname{cov}\left(\beta_{1}, \beta_{2}\right) \ldots & \operatorname{cov}\left(\beta_{1}, \beta_{P}\right) \\
\operatorname{cov}\left(\beta_{2}, \beta_{1}\right) & \operatorname{var}\left(\beta_{2}\right) \ldots & \\
\ddots & \ddots & \ddots \\
\operatorname{cov}\left(\beta_{P}, \beta_{1}\right) \ldots & \ddots & \operatorname{var}\left(\beta_{P}\right)
\end{array}\right)
$$

\subsection{Propagation of the uncertainties (Model 2)}

The substitute feature is characterized by a point $A$ and the normal vector $\vec{n}$, as shown in Figure 5 .

Let $M$ be the farthest point from the plane in the direction $\vec{n}$, and $\mathrm{m}$ the farthest point of the plane in the opposite direction. We can estimate the flatness error from the expression below:

$$
f e=|M \vec{m} \cdot \vec{n}|
$$

$$
\begin{aligned}
f e & =\left|\left(\begin{array}{l}
x_{m}-x_{M} \\
y_{m}-y_{M} \\
z_{m}-z_{M}
\end{array}\right) \cdot\left(\begin{array}{c}
n_{x} \\
n_{y} \\
n_{z}
\end{array}\right)\right| \\
& =\left|\left(x_{m}-x_{M}\right) \cdot n_{x}+\left(y_{m}-y_{M}\right) \cdot n_{y}+\left(z_{m}-z_{M}\right) \cdot n_{z}\right|
\end{aligned}
$$




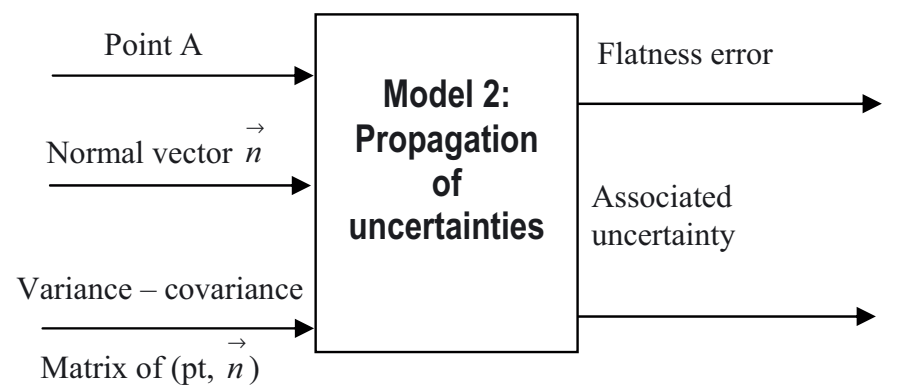

Fig. 6. Model of propagation of uncertainties.

To determine the flatness error uncertainty, we used the law of propagation of uncertainties described in the GUM [10]. This law is based on a Taylor expansion of the function $Y=f(X)$ in the first order, the variance of the output $Y$ is expressed by the following formula:

$$
\begin{aligned}
V(Y)= & \sum_{i=1}^{k}\left[\left(\frac{\partial f}{\partial X_{i}}\right)_{X=\mu}\right]^{2} V\left(X_{i}\right) \\
& +2 \cdot \sum_{i=1}^{k-1} \sum_{j>i}^{k}\left(\frac{\partial f}{\partial X_{i}}\right)_{X=\mu}\left(\frac{\partial f}{\partial X_{j}}\right)_{X=\mu} \operatorname{cov}\left(X_{i}, X_{j}\right)
\end{aligned}
$$

This law can be written in the following condensed form:

$$
V(Y)=J . \Omega . J^{T}
$$

with $J$ Jacobian matrix, $\Omega$ represents the variancecovariance matrix of the parameters.

The input and the output of the studied model are given in Figure 6.

The uncertainty of the flatness default is expressed by: $V(f e)=J . \Omega \cdot J^{T}$; and the Jacobian matrix is as follows:

$$
J=\left[\frac{\partial f e}{\partial n_{x}} \frac{\partial f e}{\partial n_{y}} \frac{\partial f e}{\partial n_{z}} \frac{\partial f e}{\partial x_{m}} \frac{\partial f e}{\partial y_{m}} \frac{\partial f e}{\partial z_{m}} \frac{\partial f e}{\partial x_{M}} \frac{\partial f e}{\partial y_{M}} \frac{\partial f e}{\partial z_{M}}\right]
$$

with:

$$
\begin{gathered}
\frac{\partial f e}{\partial n_{x}}=x_{m}-x_{M} ; \frac{\partial f e}{\partial n_{y}}=y_{m}-y_{M} ; \frac{\partial f e}{\partial n_{z}}=z_{m}-z_{M} ; \\
\frac{\partial f e}{\partial x_{m}}=n_{x} ; \frac{\partial f e}{\partial y_{m}}=n_{y} ; \frac{\partial f e}{\partial z_{m}}=n_{z} ; \\
\frac{\partial f e}{\partial x_{M}}=-n_{x} ; \frac{\partial f e}{\partial y_{M}}=-n_{y} ; \frac{\partial f e}{\partial z_{M}}=-n_{z}
\end{gathered}
$$

$\Omega$ represents the matrix of variance covariance of parameters $\vec{n}, m$ and $M$ are given bellow:

$$
\Omega=\left[\begin{array}{c}
{[\vec{n}]} \\
{[m]} \\
{[M]}
\end{array}\right],
$$

and

$$
[\vec{n}]=\left(\begin{array}{ccc}
\operatorname{var}\left(n_{x}\right) & \operatorname{cov}\left(n_{x}, n_{y}\right) & \operatorname{cov}\left(n_{x}, n_{z}\right) \\
\operatorname{cov}\left(n_{y}, n_{x}\right) & \operatorname{var}\left(n_{y}\right) \ldots & \operatorname{cov}\left(n_{y}, n_{z}\right) \\
\operatorname{cov}\left(n_{z}, n_{x}\right) & \operatorname{cov}\left(n_{z}, n_{y}\right) & \operatorname{var}\left(n_{z}\right)
\end{array}\right)
$$

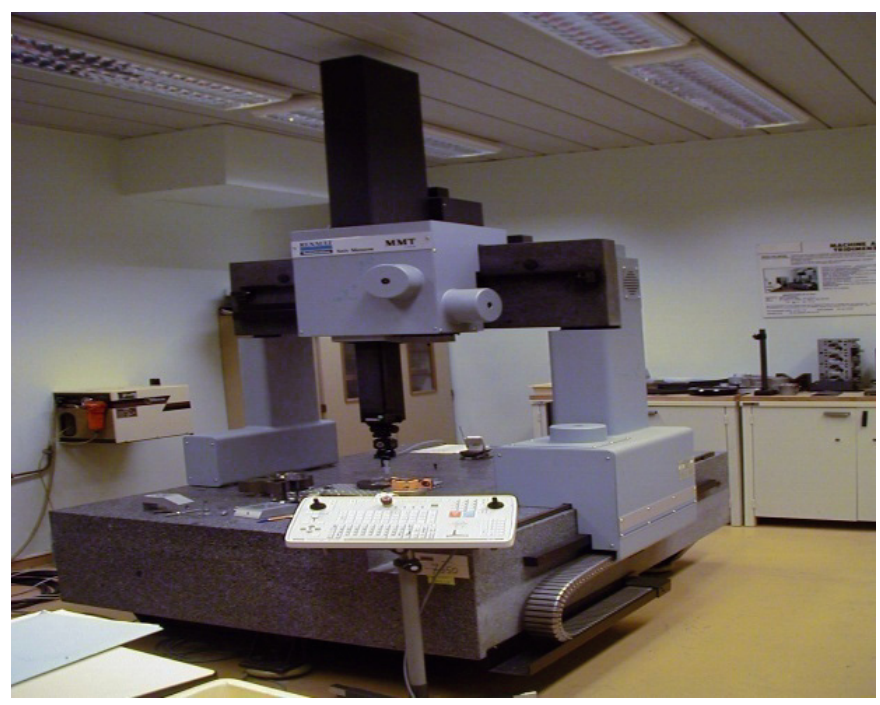

Fig. 7. CMM used.

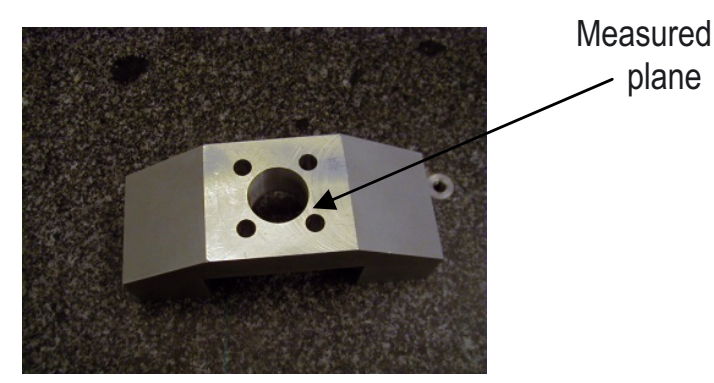

Fig. 8. The industrial part measured.

The variance-covariance matrix associated to each points $\mathrm{m}$ and $\mathrm{M}$ is given by the following formula:

$$
\left[M_{i}\right]=\left(\begin{array}{ccc}
\operatorname{var}\left(x_{i}\right) & 0 & 0 \\
0 & \operatorname{var}\left(y_{i}\right) & 0 \\
0 & 0 & \operatorname{var}\left(z_{i}\right)
\end{array}\right)
$$

\section{Experimental study}

This experimental study aims to show the effect of the number points on the flatness error and the associated uncertainties. On a coordinate measuring machine (see Fig. 7), a series of measurements of the industrial parts are made. The Machine and conditions of tests are as follows:

- CMM with mobile gantry SEIV 13-08-06. Capacity $X=1000 \mathrm{~mm}, Y=600 \mathrm{~mm}, Z=400 \mathrm{~mm}$ with probe Head PH9. Volumetric accuracy $=4.5 \mu \mathrm{m}+L / 200$, with $L$ in $\mathrm{mm}$.

- Measured parts: industrial parts.

- Type of probe: TP2.

- Medium: The machine is located in an air-conditioned room; the temperature is controlled within the range of $20 \pm 1{ }^{\circ} \mathrm{C}$. 
A. Jalid et al.: Influence of sample size on flatness estimation and uncertainty in three-dimensional measurement 102-p5

Table 1. Variation of flatness error and the associated uncertainty according to the sample size.

\begin{tabular}{cccccccccc}
\hline & \multicolumn{1}{c}{ Sample size } & & & \\
\hline & 8 & 12 & 16 & 24 & 32 & 40 & 48 & 52 & 56 \\
\hline $\begin{array}{c}\text { Flatness error (fe) } \\
\text { (in mm) }\end{array}$ & 0.00246 & 0.00491 & 0.0043 & 0.00531 & 0.00552 & 0.00657 & 0.00585 & 0.00617 & 0.00628 \\
\hline $\begin{array}{c}\text { Associated uncertainty } \\
\text { (in } \mu \mathrm{m} \text { ) }\end{array}$ & 3,8244 & 4,0848 & 3,7723 & 3,7738 & 3,7726 & 3,7326 & 3,7222 & 3,6570 & 3,6401 \\
\hline
\end{tabular}

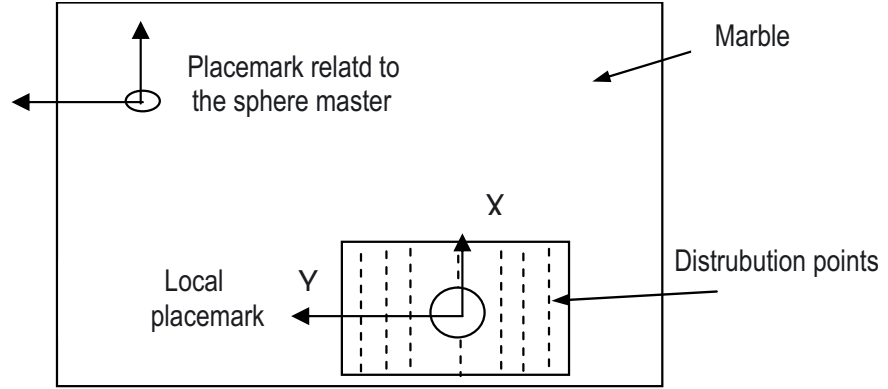

Fig. 9. Distrubution points taken from the plane.

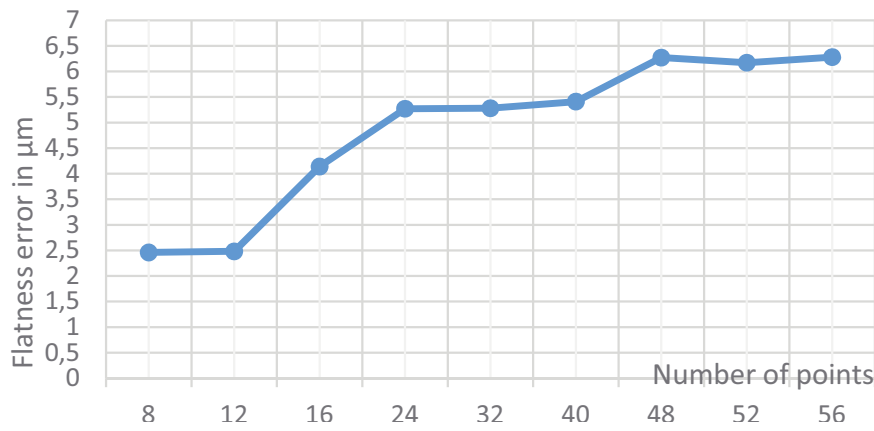

Fig. 10. Variation of flatness error according to the sample size.

An industrial part was measured on the machine as shown in Figure 8. We collected from a plane feature 56 points. The coordinates of measured points are relatively given to the local placemark $(\mathrm{O}, X, Y, Z)$ related to the measured part, see Figure 9.

From this cloud of points, we constructed samples of 8 , $12,16,24,32,36,40,48,52$ and 56 points, distributed over the surface.

\subsection{Effect of sample size on flatness and the associated uncertainty}

For each sample points, Model 1 and Model 2 are applied to estimate the flatness error and the associated uncertainty. The results found are given in Table 1 .

These results may be plotted on graphs as shown in Figures 10 and 11.

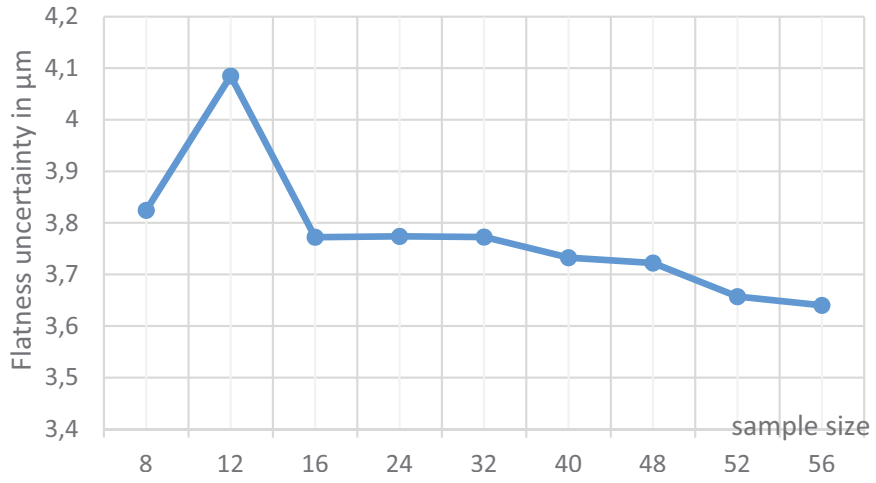

Fig. 11. Variation of the associated uncertainty according to the sample size.

\subsection{Analysis of results}

In Figure 11 above, we can see that the number of points affects the flatness error. When this number increases, the flatness defect slightly increases and stabilizes thereafter at a value that seems to be the most probable. However, in Figure 12 when this number increases, the flatness defect uncertainty decreases slightly to stabilize asymptotically, in our study it stabilizes between 3.6 and 3.7 microns. This reduction is in agreement with equation (4) that gives the residual variance, which is inversely proportional to the number of points $n$.

\section{Conclusion}

The present study shows the influence of the number of points collected on a plane surface on the flatness deviation and the associated uncertainty. This number must be selected correctly; the sample size must give a good representation of the surface while respecting the constraint on the measurement time. The reduction in time reduces the control cost.

In this study we have shown the influence of the sample size on flatness and the associated uncertainty. The results found can be summarized as follows:

- The more we increase the number of points, the representation of the measured element is better.

- The number of points increases slightly the flatness error which converges to a value that seems to be most probable. 
- The number of points reduces the flatness uncertainty, and therefore a reduced number of points overestimates the uncertainty which may lead to an erroneous conformity decision.

- The choice of the sample size must be optimal; this number must strike a compromise between the desired accuracy and time measurement. In our case, this number is about 16 points.

To meet the normative guidelines, [10] requires that each measure must be provided with its uncertainty. This study contributes to assess the uncertainty measurement and demonstrates the impact of the sample size taken on the feature of part on the flatness error and the associated uncertainty.

\section{References}

1. P. Bourdet, Contribution à la mesure tridimensionnelle : Modèle d'identification géométrique ; correction des machines à mesurer ; métrologie fonctionnelle des pièces mécaniques, Ph.D. thesis, University of Nancy 1, France (1988)

2. L. Mathieu, A. Castang, Contrôle des spécifications par zone de tolérance (ISO 1101): Quantification des erreurs causées par les méthodes de traitement des points mesurés, Proceedings of international congress of metrology 1995, Nimes, France

3. K. Carr, P. Ferreira, Verification of form tolerances. Part I: Basic issues, flatness and straightness, Precis. Eng. 17, 131-143 (1995)

4. T. Kanada, S. Suzuki, Evaluation of minimum zone flatness by means of nonlinear optimization techniques and its verification, Precis. Eng. 15, 93-99 (1993)

5. R. Raghunandan, P. Venkateswara Rao, Selection of sampling points for accurate evaluation of flatness error using coordinate measuring machine, J. Mater. Process. Technol. 202, 240-245 (2008)
6. R. Raghunandan, P. Venkateswara Rao, Selection of an optimum sample size for flatness error estimation while using coordinate measuring machine, Int. J Machine Tools Manufact. 47, 477-482 (2007)

7. Jyunping Huang, An efficient approach for solving the straightness and the flatness problems at large number of data points, Computer-Aided Design 35, 15-25 (2003)

8. B. Gapinski, M. Grzelka, M. Rucki, The accuracy analysis of the roundness measurement with coordinate measuring machines, XVIII IMEKO world congress, September 2006, Rio de Janeiro, Brazil

9. G.L. Samuel, M.S. Shunmugam, Evaluation of straightness and flatness error using computational geometric techniques, Computer-Aided Design 31, 829-843 (1999)

10. ISO/IEC Guide 98-3:2008, Uncertainty of measurement Part 3: Guide to the expression of uncertainty in measurement (GUM, 1995)

11. Norme ISO 1101:2004, Geometrical product specifications (GPS)- Geometrical tolerancing - Tolerances of form, orientation, location and run-out, 2004

12. A. Jalid, S. Hariri, J.P. Senelaer, A. El gharad, Un algorithme pour l'estimation des paramètres de courbes (ou surfaces) et leurs incertitudes en mesure 3D, Proceedings of international conference on Integrated design and production (CPI'2007), 22-24 octobre, 2007, Rabat, Maroc

13. K. Levenberg, A method for the solution of certain problems in least squares, Quart. Appl. Math. 2, 164-168 (1944)

14. D. Marquardt, An algorithm for least-squares estimation of nonlinear parameters, SIAM J. Appl. Math. 11, 431-441 (1963)

15. J. Nocedal, S.J. Wright, Numerical Optimization, Springer, New York (1999)

16. P.T. Boggs, H. Byrd Richard, E.R. Janet, R.B. Schnabel, Users reference guide for ODRPACK version 2.01, Software for weightes orthogonal distance regression (1992)

17. P.T. Boggs, R.H. Byrd, B. Schnabel Robert, A stable and efficient algorithm for nonlinear orthogonal distance regression, SIAM J. Sci. Statist. Comput. 1052-1078 (1987) 